\title{
Cytokine concentrations in bronchoalveolar lavage fluid (BALF) from children with severe community-acquired pneumonia (SCAP) from Shanghai
}

\author{
bingru yin ${ }^{1}$, Min $\mathrm{Lu}^{2}$, Guodong Ding ${ }^{1}$, Na Dong ${ }^{1}$, Xiaoyan Dong ${ }^{1}$, and Haoxiang Gu ${ }^{1}$ \\ ${ }^{1}$ Shanghai Children's Hospital \\ ${ }^{2}$ Shanghai United Family Hospital
}

November 16, 2021

\begin{abstract}
Background: It has been well known that overreaction of host immune systems plays a critical role in severe communityacquired pneumonia (SCAP). However, few of previous studies focused on the association between cytokine concentrations in bronchoalveolar lavage fluid (BALF) and SCAP. Object: We examined cytokine concentrations in BALF from children with SCAP and explored predictive value of cytokine concentrations for SCAP. Method: It was a retrospective study. A total of 100 children with SCAP who underwent parallel bronchoalveolar lavage between July 2017 and June 2019 were enrolled. 100 patients with mild community-acquired pneumonia (MCAP) who were admitted to the hospital were matched based on age and sex during the same time period. Basic clinical information were collected. Flow cytometry was used to determine the cytokine concentrations in BALF. Receiver operating characteristic curve was used to analyze their predictive value for SCAP. Result: A total of 106 males and 94 females were included in this study. The results showed that the CRP, PCT, ESR, LDH, and D-dimer were significantly increased in the SCAP group. The SCAP patients also had longer fever duration, hospitalization stays, and higher hospitalization costs. The IL-5,IL-17A,IL-18,and TnF- $\alpha$ in BALF of SCAP group were significantly higher than those in MCAP group. ROC curve analysis demonstrated AUC of those cytokines were all among 0.5 0.7. Conclusion: The IL-5,IL-17A,IL-18, and TnF- $\alpha$ in BALF of children with SCAP group were highe. However, the efficacy of those cytokines in the clinical diagnosis of SCAP is not excellent to be used as a predictor.
\end{abstract}

\section{INTRODUCTION}

Community-acquired pneumonia is the most common cause of hospitalization in children. Severe pneumonia is one of the leading causes of death in children under 5 years old in China ${ }^{[1]}$. Common pathogens of pneumonia include Streptococcus pneumoniae, mycoplasma, adenovirus, etc ${ }^{[2-5]}$. The cytotoxicity and inflammation is a crucial part of the pathogenesis in severe lung infection ${ }^{[6]}$. Therefore, early identification of patients with severe community-acquired pneumonia(SCAP), timely treatment, are crucial to block the disease process and improve the prognosis of children. In pathogen-caused pneumonia, such as Escherichia coli pneumonia, Streptococcus pneumoniae pneumonia, Klebsiellapneumoniae pneumonia, interleukin-6 (IL6) and interleukin-10 (IL-10) were found important in inflammation ${ }^{[7-9]}$. Bronchoscopy and bronchoalveolar lavage are important methods for the diagnosis and treatment of pneumonia in children ${ }^{[10]}$. Cytokines are important mediators in both lung defense and inflammation ${ }^{[11]}$. Elevated serum pro-inflammatory cytokines, such as IL-6, have been used in prognostic assessment and as a marker for the severity of the infection ${ }^{[12-13]}$. At present, few researchs of cytokines concentrations in BALF have been reported. In this study, we aimed to investigate the efficacy of cytokine concentrations in bronchoalveolar lavage fluid (BALF) in the clinical diagnosis of SCAP.

\section{METHODS}




\section{Study Population}

A total of 100 children with SCAP who underwent parallel bronchoalveolar lavage between July 2017 and June 2019 were enrolled in our department. 100 patients with mild community-acquired pneumonia (MCAP) who were admitted to the hospital were matched based on age and sex during the same time period (within 2 weeks). When the patients were [?]1 year old, the age of the matched patients was within \pm 6 months. When the patients were $<1$ year old, the age of the matched patients was within \pm 3 months. Written informed consent was obtained from each pediatric patient's parents before performing bronchoscopy in clinical practice. The guardians signed informed consent before the samples were collected. Being a retrospective study, no consent was required for the analysis of the data retrieved from medical records. The research protocol for this study was approved by the Medical Ethics Committee of Shanghai Children's Hospital, Shanghai Jiao Tong University.

\section{Diagnosis}

The MCAP and SCAP were defined based on the criteria described ${ }^{[14-16]}$. The mild group defined as fever $<$ 38.5 at any age, tachypnea but respiratory rate $<70$ breaths $/ \mathrm{min}$ at age $<3$ years old or $<50$ breaths $/ \mathrm{min}$ at age $>3$ years old, normal food-intake, no dehydration. The severe group defined as fever over 38.5 at any age, breathless with respiratory rate of 70 breaths/min at age $<3$ years old or $<50$ breaths $/$ min at age 3 years old (excluding the reasons of fever and cry), cyanosis $(\mathrm{SpO} 2[?] 92 \%)$, flaring of the nares, marked retractions, anorexia, and dehydration.

\section{Bronchoscopy and BALF Procedure}

The indications for bronchoscopy were persistent when the temperature continued to fluctuate for greater than 5 days after conventional treatment and the clinical symptoms were not obviously relieved or radiological abnormalities (atelectasis and consolidation of lung fields) were suspected. Flexible fiberoptic bronchoscopy with BALF was performed between the 2 nd and $7^{\text {th }}$-day post-admission following the guidelines described previously ${ }^{[17]}$. A 2.8-mm pediatric flexible bronchoscope (Olympus BF-XP60 or BF-XP40; Olympus, New Hyde Park, NY) was used for young children, and a 4.0-mm flexible bronchoscope (Olympus BF-3C30) for older children depending on the age and the body weight of the children. Supplemental oxygen was administered during the procedure. Transcutaneous oxygen saturation and pulse rate (Pulsoxymetr 400HS, TRIDENT MED, Poland) were continuously monitored during the bronchoscopy.

Midazolam $(0.1-0.2 \mathrm{mg} / \mathrm{kg})$ intravenous injection for sedation and aerosolized lidocaine spraying on the throat for local anesthesia was used 5-10 min before bronchoscope insertion. Topical anesthesia of the upper and lower airways was performed by dripping $2 \%$ lidocaine during flexible bronchoscopy. BALF was performed either in an area most prominently affected on the chest radiology. Boluses of $1 \mathrm{ml} / \mathrm{kg}$ of sterile, nonbacterialstatic saline at room temperature were instilled through the instrumentation channel. The BALF was gently aspirated immediately after each aliquot and collected in a sterile container and immediately placed in 4 .

\section{Processing and Analysis of the BALF}

BALF was immediately aspirated into a sterile specimen container using a wall suction pressure of $100 \mathrm{~mm}$ Hg. A Cytometric Bead Array (CBA) specific for cytokines (BD Biosciences, San Diego, CA) was used to measure the concentrations of IL-17A, IL-18, IL-5, IL-12p70,

IL-8, IL-1 $\beta$, IL-2, IL-10, IL-6, IL-4, TNF- $\alpha$, and $\gamma$-TNF, according to the manufacturer's instructions. Briefly, microbead populations with distinct fluorescence signals were coated with capture antibodies specific for each cytokine. The capture beads, phycoerythrin-conjugated detection antibodies, and test samples (recombinant standards used to generate a standard curve) were incubated at room temperature, and the BALF of children was diluted $1: 4$ in assay diluent and pelleted by centrifugation $(10,000 \mathrm{rpm} \times 5 \text { minutes })^{[18]}$. After careful aspiration of the supernatant, the beads were resuspended in $300 \mu \mathrm{l}$ wash buffer, vortexed briefly, and analyzed on a BD FACScan using BD CBA analysis software (BD biosciences). For absolute quantification, the assay was calibrated with dilutions of cytokines chimera. 


\section{Statistical Analysis}

The database was built using EpiData 3.1 software, and all recorded data were statistically analyzed using SPSS 22.0 statistical software. All data were tested for normal distribution, in which cytokines were nonnormally distributed. In descriptive statistics, non-normally distributed data is represented as median (25-75 quartile spacing). Differences between the severe and non-severe groups were compared by the Mann-Whitney $\mathrm{U}$ test. Statistically significant results for $\mathrm{P}$ values are indicated in bold. The ROC curve model was used to analyze the value of cytokine and cell level in the clinical diagnosis of the severity of the disease. The area under each curve (AUC) was compared. The AUC ranged from 0.5 to 0.7 , indicating a lower diagnostic efficiency. The AUC was 0.7-0.9. The prompt has a certain diagnostic efficiency, and above 0.9 indicates higher diagnostic efficiency. $P$ value $<0.05$ was considered statistically significant.

\section{RESULTS}

\section{Patient characteristics}

A total of 100 patients in the SCAP group were included in this study, 100 patients in the MCAP group were matched based on age and sex. A total of 106 males and 94 females were included in this study, with a male-to-female ratio of 1.13:1.

The ages of 18 cases (9\%) were within 1 year old, 41 cases (20.5\%) were aged 1-3 years, 74 cases (37\%) were aged 3-6 years, and 67 cases (33.5\%) were aged 6 years or older. Comparing the serum inflammation indicators of the two groups, results showed that the CRP, PCT, ESR, and LDH in the SCAP group were significantly increased, and the difference were statistically significant (Table 1). In addition, comparing the D-dimer of the two groups also indicated that the D-dimer of the SCAP group was higher, and the difference was also statistically significant $(\mathrm{p}=0.000)$.

Comparisons of fever duration, hospitalization stays, and hospitalization expenditures between the two groups of children revealed that SCAP patients have longer fever duration and hospitalization stays, and hospitalization costs were also higher. The difference was statistically significant $(p=0.000, p=0.002, p$ $=0.008$, respectively). Comparing the composition of the pathogens between the two groups, the results showed that $71 \%$ of patients in the MCAP group had positive pathogens. In contrast, $89 \%$ of patients in the SCAP group had positive pathogens. Mycoplasma infections were the most common pathogens in both groups followed by coinfections. No significant difference in the composition of pathogens was noted between the two groups.k

Comparison of concentrations of cytokines in alveolar lavage fluid. The concentrations of cytokines in the SCAP group and the MCAP group were compared. The concentrations of cytokines (IL-5,IL-17A,IL$18, \mathrm{TnF}-\alpha)$ in the SCAP group were higher than in the MCAP group, and the difference were statistically $\operatorname{significant}(Z=2.513, P=0.012 ; Z=2.404, P=0.016 ; Z=2.982, P=0.003 ; Z=2.689, P=0.007)$. No significant difference were found in concentrations of cytokines(IL-8 、IL-4、IL-1 $\beta$ IL-2、IL-12p70、IL-6 、IL-10、 $\gamma$ TNF)between the severe and mild groups(Table 2) .

The ROC curve of cytokine levels in alveolar lavage fluid to predict children with SCAP. To further explore the predictive values of the aforementioned cytokines as biomarkers for SCAP, ROC curves were performed and the cut-off scores with maximum sensitivities and specificities were determined. Analyses of these ROC curves showed that when the cutoff values for IL-5, IL-17A, IL-18, and TnF- $\alpha$ were set at $5.045 \mathrm{pg} / \mathrm{mL}, 0.380 \mathrm{pg} / \mathrm{mL}, 4.970 \mathrm{pg} / \mathrm{mL}$, and $0.105 \mathrm{pg} / \mathrm{mL}$, respectively, the sensitivity and specificity in differentiating SCAP from MCAP were $22 \%$ and $91 \%, 20 \%$ and $93 \%, 68 \%$ and $59 \%, 40 \%$ and $80 \%$, respectively. Receiver operating characteristic (ROC) curve analysis demonstrated an area under the curve (AUC) of 0.57 (CI 0.491-0.650) for IL-5, 0.559 (CI 0.480-0.693) for IL-17A, 0.617 (CI 0.539-0.696) for IL-18, and 0.589 (CI 0.510-0.668) for TNF- $\alpha$. We found that the AUC for IL-5,IL-17A,IL-18,and TnF- $\alpha$ were above 0.5 and below 0.7 in ROC curve analysis, the efficacy of cytokines in the clinical diagnosis of SCAP is not excellent to be used as a predictor. (Table 3; Figure 1) .

\section{Discussion}


Severe pneumonia is one of the leading causes of death in children under 5 years old in China ${ }^{[1]}$. So early identification of severe pneumonia is very important. In this study, 100 patients who underwent fiberoptic bronchoscopy due to SCAP were enrolled, and 100 patients with MCAP were matched based on age and sex. The inflammation makers (WBC, CRP, ESR, PCT, Fer, etc.), fever duration, hospitalization stays, and hospitalization expenses were compared. At the same time, the cytokines in the alveolar lavage fluid of the two groups were compared to explore the prediction factors of SCAP.

Comparing the serum inflammation markers of the two groups, the results showed that the CRP, PCT, ESR, and LDH levels of the SCAP group were significantly higher, and the difference was statistically significant. This finding suggests that patients in the SCAP group have a stronger inflammatory response. These results are similar to those of previous studies ${ }^{[19,20]}$. The results of the study show that patients with severe pneumonia have abnormal blood coagulation after infection, causing blood hypercoagulability, and a risk of complicated thrombosis is noted ${ }^{[21,22]}$. D-dimer levels were significantly increased in the SCAP group. These findings suggest that patients with SCAP need to be alert to the state of hypercoagulable blood to avoid thrombosis.

The results also suggested that SCAP patients had longer fever duration and hospitalization stays, and higher hospitalization costs. The difference were statistically significant $(p=0.000, p=0.002, p=0.008)$. It suggests that SCAP patients are seriously ill, having a longer fever duration and a longer hospital stays, and spending more, which are a serious economic burden for parents and society.

Severe pneumonia is the first cause of death for children under 5years old in China ${ }^{[1]}$. Therefore, it is important to find more effective biomarkers to diagnose severe pneumonia. In this study, we explored the value of concentrations of cytokines in BLAF in the diagnosis of SCAP. The results revealed that the concentrations of IL-5,IL-17A,IL-18, and TnF- $\alpha$ in BALF were higher among those with SCAP than among those with MCAP.

At present, lots of studies have shown that serum cytokines are associated with respiratory infections. It has previously been shown that children with a laboratory-confirmed influenza A (2009 H1N1) virus pneumonia had higher IL-5 concentrations compared to children without a lower respiratory tract infection ${ }^{[23-25]}$. Other studies supported that in children elevated serum IL-5 levels have also been found in wheezing caused by a rhinovirus or respiratory syncytial virus infection ${ }^{[26]}$.

IL-17A,IL-18 and TnF- $\alpha$ are pro-inflammatory cytokines ${ }^{[27,28,29]}$.

Cytokines play an important role in the infection of different pathogens. In P. aeruginosa chronic airway infection, a study identifies IL-17-mediated response as a negative regulator of host tolerance during ${ }^{[30]}$. In S. aureus infections, these novel findings reveal that S. aureus activates the NLRC4 to dampen IL-17Adependent neutrophil accumulation through induction of necroptosis and IL-18 ${ }^{[28]}$. Meta-analysis showed that serum TNF- $\alpha$ and IFN-c levels were significantly elevated and unchanged, respectively, in pediatric $\mathrm{MPP}^{[31]}$.

In addition, studies have shown that the more severe the infection is, the higher the cytokine concentrations are. A study among 45 mechanically ventilated, intensive care unit (ICU)-treated severe community-acquired pneumonia (SCAP) patients indicated SCAP patients with viral etiology have higher IL-5 levels ${ }^{[32]}$. Circulating and alveolar IL-17A concentrations are closely linked with alveolar neutrophilic inflammation in ARDS. Elevated circulating IL-17A is associated with increased organ dysfunction in patients with ARDS ${ }^{[33]}$. In our research, we found similar results. It suggests that after the lungs are infected with pathogenic bacteria, the lung tissues are locally damaged, forming an immune response, leading to increased cytokine levels.

We further analyzed the value of the cell level in the clinical diagnosis of the severity of the disease in children through the ROC curve model, and compared the area under the curve (AUC) of each factor, and found that most of the AUC are between 0.5-0.7. The efficacy of those cytokines in the clinical diagnosis of SCAP is not excellent to be used as a predictor. The expression level of serum IL-18 was significantly higher in the non-survival group than that in the survival group $(\mathrm{P}<.05)$. Based on the ROC curve, the sensitivity and 
specificity of IL-18 as a predictor of prognosis at a cutoff of $509.5 \mathrm{pg} / \mathrm{mL}$ were $88 \%$ and $82 \%$, respectively, and the area under the curve (RUC) was $0.84(\mathrm{P}<.05)$. In summary, our finding suggested that serum IL-18 might serve as a prognostic biomarker in $\operatorname{ARDS}^{[29]}$. Makabe et $\mathrm{al}^{[34]}$ has reported that IL-18 expression level in the serum was significantly higher in the non-survivors of ARDS patients. It is different from our research results. It may be related to the disease severity of the patients included in the study.

\section{Conclusions}

Our findings suggested that the concentrations of these cytokines in BALF of children were higher among those with SCAP than among those with MCAP, however, the efficacy of those cytokines in the clinical diagnosis of SCAP is not excellent to be used as a predictor.

AcknowledgmentsThe work described in this paper was fully supported by a grant from the Clinical Research and Cultivation Program of Shanghai Children's Hospital affiliated to Shanghai Jiaotong University (No.2020YLYM03).

\section{References}

1.Song P, Theodoratou E, Li X, et al. Causes of death in children younger than five years in China in 2015: an updated analysis.J Glob Health. 2016, 6(2):020802. doi: 10.7189/jogh.06.020802.

2.Rudan I, Boschi-Pinto C, Biloglav Z, Mulholland K, Campbell H. Epidemiology and etiology of childhood pneumonia. Bull World Health Organ. 2008. 86(5): 408-16.

3.Ning G, Wang X, Wu D, et al. The etiology of community-acquired pneumonia among children under 5years of age in mainland China, 2001-2015: A systematic review. Hum VaccinImmunother. 2017. 13(11): $2742-2750$.

4.Tramper-Stranders GA. Childhood community-acquired pneumonia: A review of etiology-and antimicrobial treatment studies. PaediatrRespir Rev. 2018. 26: 41-48.

5.ain S, Williams DJ, Arnold SR, et al. Community-acquired pneumonia requiring hospitalization among U.S. children. N Engl J Med. 2015. 372(9): 835-45.

6.Waites KB, Talkington DF. Mycoplasma pneumoniae and its role as a human pathogen.ClinMicrobiol Rev 2004;17:697-728.

7.Quinton LJ, Jones MR, Robson BE, Simms BT, Whitsett JA, Mizgerd JP. Alveolar epithelial STAT3, IL-6 family cytokines, and host defense during Escherichia coli pneumonia. Am J Respir Cell MolBiol 2008;38:699-706.

8.Lieberman D, Livnat S, Schlaeffer F, Porath A, Horowitz S, Levy R. IL-1beta and IL-6 in communityacquired pneumonia: Bacteremic pneumococcal pneumonia versus Mycoplasma pneu-moniae pneumonia. Infection 1997;25:90-94.

9.Poe SL, Arora M, Oriss TB, Yarlagadda M, Isse K, Khare A, Levy DE, Lee JS, Mallampalli RK, Chan YR, et al. STAT1-regulated lung MDSC-like cells produce IL-10 and efferocytose apoptotic neutrophils with relevance in resolution of bacterial pneumonia. Mucosal Immunol 2013;6:189-199.

10.Duarte JC, Cordeiro CR, Ferreira AJ. Role of flexible bronchoscopy in non-resolving community-acquired pneumonia - A retrospective analysis. Rev Port Pneumol (2006), 2017;23(3):168-169.

11.Tumgor G, Celik U, Alabaz D, et al. Aetiological agents, interleukin-6, interleukin-8 and CRP concentrations in children with community- and hospital-acquired pneumonia. Ann Trop Paediatr 2006;26:285-291.

12.

13. 
14.Subspecialty Group of Respiratory Diseases, The Society of Pediatrics, Chinese Medical Association The Editorial Board, Chinese Journal of Pediatrics. [Guidelines for management of community acquired pneumonia in children(the revised edition of 2013) (II)]. ZhonghuaErKeZaZhi. 2013. 51(11): 856-62.

15.Subspecialty Group of Respiratory Diseases, The Society of Pediatrics, Association CM, Editorial Board, Chinese Journal of Pediatrics. Guidelines for management of community acquired pneumonia in children (the revised edition of 2013) (I)]. ZhonghuaErKeZaZhi. 2013. 51(10): 745-52.

16. Harris M, Clark J, Coote N,et al. British Thoracic Society guidelines for the management of community acquired pneumonia in children: update 2011.Thorax. 2011 Oct;66Suppl 2:ii1-23. doi: 10.1136/thoraxjnl-2011-200598.

17.Chernick V, Boat TF, Wilmott RW, Bush A, Deterding RR, Ratjen FA. Kendig's disorders of the respiratory tract in children.(7th edition). Philadelphia: 2006.

18.Hirani R, Dean MM, Balogh ZJ, et al. Donor white blood cell survival and cytokine profiles following red blood cell transfusion in Australian major trauma patients. MolImmunol. 2018. 103: 229-234.

19. Torres A, Sibila O, Ferrer M, et al. Effect of corticosteroids on treatment failure among hospitalized patients with severe community-acquired pneumonia and high inflammatory response: a randomized clinical trial. JAMA, 2015,313(7):677-86.

20.Principi N, Esposito S. Biomarkers in Pediatric Community-Acquired Pneumonia. Int J Mol Sci, 2017,18(2):447.

21.Ge YL, Liu CH, Wang N, Xu J, et al. Elevated Plasma D-Dimer in Adult Community-Acquired Pneumonia Patients is Associated with an Increased Inflammatory Reaction and Lower Survival.Clin Lab. 2019;65(1).

22. Cerda-Mancillas MC, Santiago-Germán D, Andrade-Bravo B, et al. D-dimer as A Biomarker of Severity and Adverse Outcomes in Patients with Community Acquired Pneumonia.Arch Med Res. 2020;51(5):429435.

23.t al.

24.

25.

26 . et al.

27. Kumar S, Joos G, Boon L, et al. Role of tumor necrosis factor- $\alpha$ and its receptors in diesel exhaust particle-induced pulmonaryinflammation. Sci Rep. 2017 Sep 14;7(1):11508. doi: 10.1038/s41598-01711991-7.

28. Sagar Paudel, Laxman Ghimire, Liliang Jin, et al. NLRC4 suppresses IL-17A-mediated neutrophildependent host defense through upregulation of IL-18 and induction of necroptosis during Gram-positive pneumonia. Mucosal Immunol. 2019 Jan; 12(1): 247-257.

29. Guangsu Dong, Fei Wang, Liang Xu, et al. Serum interleukin-18

A novel prognostic indicator for acute respiratory distress syndrome. Medicine (Baltimore). 2019 May; 98(21): e15529.

30. Nicola Ivan Lorè, Cristina Cigana, Camilla Riva,et al. IL-17A impairs host tolerance during airway chronic infection by Pseudomonas aeruginosa. Sci Rep. 2016; 6: 25937.

\section{Hosted file}

Table1.docx available at https://authorea.com/users/446342/articles/545605-cytokineconcentrations-in-bronchoalveolar-lavage-fluid-balf-from-children-with-severe-communityacquired-pneumonia-scap-from-shanghai 


\section{Hosted file}

Table2.docx available at https://authorea.com/users/446342/articles/545605-cytokineconcentrations-in-bronchoalveolar-lavage-fluid-balf-from-children-with-severe-communityacquired-pneumonia-scap-from-shanghai

\section{Hosted file}

Table3.docx available at https://authorea.com/users/446342/articles/545605-cytokineconcentrations-in-bronchoalveolar-lavage-fluid-balf-from-children-with-severe-communityacquired-pneumonia-scap-from-shanghai
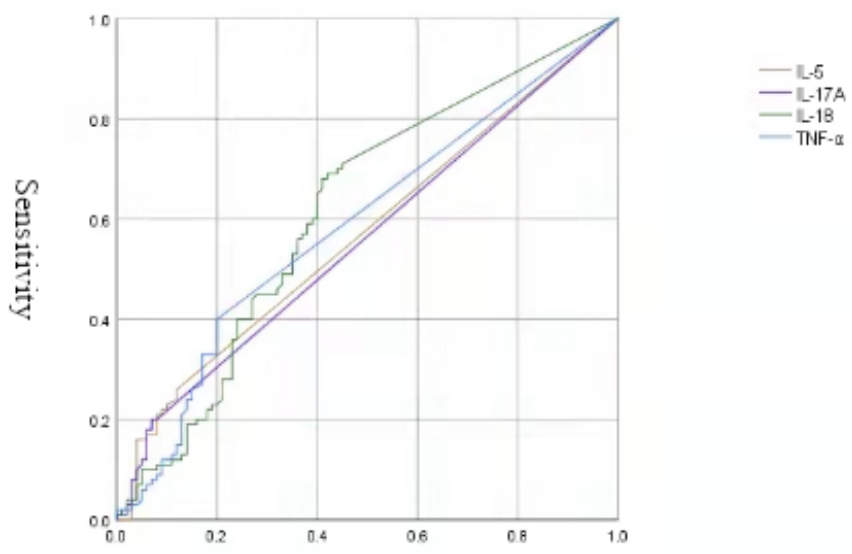

1-Specificity

Figure 1 The ROC curve of cytokine levels in alveolar lavage fluid to predict children with SCAP. 